\title{
Severidad del daño causado por los incendios forestales en los bosques remanentes de Nothofagus alessandrii Espinosa en la Región del Maule de Chile
}

\section{Fire severity damages caused on Nothofagus alessandrii forest on the Maule Region of Chile}

\author{
Diego Valencia ${ }^{1 *}$, Jorge SaAvedra ${ }^{2}$, Jordi Brull² \& Rómulo Santelices ${ }^{3}$
}

\begin{abstract}
1Departamento de Planificación de Áreas Silvestres Protegidas, Gerencia de Áreas Silvestres Protegidas, Corporación Nacional Forestal (CONAF), Bulnes 285, Santiago, Chile.

${ }^{2}$ Departamento de Control de Incendios Forestales, Gerencia de Prevención de Incendios Forestales, Corporación Nacional Forestal (CONAF), Bulnes 285, Santiago, Chile.

${ }^{3}$ Centro de Desarrollo Para el Secano Interior, Facultad de Ciencias Agrarias y Forestales, Universidad Católica del Maule, Casilla 617, Talca, Chile.

*diego.valencia@conaf.cl
\end{abstract}

\begin{abstract}
A forest fire in 2017 consumed about 184,000 hectares among which an important part of Nothofagus alessandrii forests, which is also an endangered species, was affected. Sentinel II images and the Normalized Burn Ratio (dNBR) indices were used to evaluate the fire severity on $N$. alessandrii forests. Results show that 172 hectares were affected by the fire $(55 \%$ of the known remaining forests). High severity and Moderate-high severity burn surface was 89 hectares ( $28 \%$ of the total burned) and this would be the most difficult area to be recovered by the system.
\end{abstract}

En la zona mediterránea de Chile se concentra el mayor endemismo asociado a grado de amenaza (Myers et al. 2000) y la mayor biodiversidad amenazada, con una rica flora y fauna autóctona (Olivares et al. 2005). Durante los meses de enero y febrero de 2017 , en parte importante de esta zona ocurrieron incendios forestales de gran magnitud e intensidad que impactaron, entre otros aspectos, los ecosistemas naturales que son de una alta relevancia global para la conservación de la biodiversidad. Algunos de estos ecosistemas se presentan en superficies reducidas, con niveles de perturbación importantes y con hábitats altamente fragmentados (Bustamante \& Grez 1995). Un ejemplo de ello son los bosquetes remanentes de Nothofagus alessandrii Espinosa (ruil) (Nothofagaceae).

$N$. alessandrii es una especie con una distribución natural limitada a una franja corta en la Cordillera de la Costa de la Región del Maule, de aproximadamente $100 \mathrm{k}$ de extensión latitudinal y en precario estado de conservación (Santelices et al. 2012). Actualmente, el ruil está declarado como un Monumento Natural (D.S. 13/1995 del Ministerio de Agricultura) y en Chile se le clasifica como una especie rara y en peligro de extinción (D.S. 151/2007 del Ministerio Secretaría General de la Presidencia). A nivel internacional, la Unión Internacional para la Conservación de la Naturaleza (UICN) la cataloga como una especie en peligro de extinción (Barstow et al. 2017). Las poblaciones remanentes tienen una distribución discontinua, se encuentran muy fragmentadas y rodeadas por plantaciones de especies alóctonas y son exitosamente invadidas por Pinus radiata D. Don (Bustamante \& Grez 1995, Bustamante \& Castor 1998). De acuerdo a lo reportado por Santelices et al. (2012), en un periodo de 17 años (entre 1991 y 2008) la superficie de los bosques de $N$. alessandrii disminuyó en 42 ha (equivalente a una tasa de deforestación de un $0,74 \%$ ), existiendo un remanente de aproximadamente 314 hectáreas distribuidas en 15 sectores (Tabla 1).

Durante el verano de 2017 en la zona centro-sur de Chile se desarrollaron los más grandes incendios registrados en la historia del país (De la Barrera \& Ruiz 2017). En este contexto, el denominado "Las Máquinas" (Fig. 1) fue uno de los de mayor impacto y afectó diferentes ecosistemas en las comunas de Empedrado, Cauquenes, Constitución y San Javier. El incendio comenzó el día 20.01.2017 a 12 $\mathrm{k}$ al norte de la ciudad de Cauquenes y se controló el día 09.02.2017, alcanzando una superficie de aproximadamente 184.000 ha. El mayor crecimiento del incendio se produjo entre los días 25 y 26 de enero en los que aumentó 116.000 ha. Este crecimiento se explica por las elevadas velocidades de propagación e intensidades del fuego que se produjeron 
como consecuencia de un escenario complejo conformado por varios factores como la meteorología extrema, la sequía acumulada, la alta carga de combustible disponible y la simultaneidad-magnitud de los incendios (CONAF 2017).

El objetivo de este trabajo es evaluar preliminarmente el daño provocado por los incendios forestales ocurridos en la zona central del país en los bosquetes de $N$. alessandrii. Esta información debe ser considerada sólo como una aproximación del daño producido por el fuego a este ecosistema tan singular y debe ser contrastada con información de campo.

La severidad de los incendios corresponde a una métrica del impacto de los incendios que está definida operacionalmente por la pérdida de la materia orgánica por encima y debajo del suelo (Keeley 2009). Tal severidad ha sido cuantificada mediante aproximaciones con imágenes satelitales utilizando el índice differenced Normalized Burn Ratio (dNBR) (Key \& Benson 2006, Miller \& Thode 2007, Escuin et al. 2008, Navarro et al. 2017). Este índice relaciona las bandas del espectro electromagnético mediante la diferencia entre el infrarrojo cercano (NIR) y el infrarrojo lejano (SWIR), en forma previa y posterior al incendio y su cálculo se puede realizar mediante las fórmulas que se muestran a continuación.

$$
\begin{aligned}
& N B R=\frac{(N I R-S W I R)}{(N I R+S W I R)} \\
& d N B R=\text { NBRprevio }- \text { NBRposterior }
\end{aligned}
$$

TABLA 1. Localización y distribución temporal de los bosquetes de Nothofagus alessandrii en los años 1991 y 2008 (Santelices et al. 2012).

\begin{tabular}{|c|c|c|c|c|c|}
\hline \multirow[t]{2}{*}{ Comuna } & \multirow[t]{2}{*}{ SECTOR } & \multicolumn{2}{|c|}{$\begin{array}{c}\text { LOCALIZACIÓN } \\
(\text { CoORDENADAS UTM })^{*}\end{array}$} & \multicolumn{2}{|c|}{ SUPERFICIE (HA) } \\
\hline & & NoRTE & Este & AÑo 1991 & AÑo 2008 \\
\hline \multirow[t]{4}{*}{ Curepto } & Huelón (9) & 766973 & 6113298 & 10,6 & 7,6 \\
\hline & Catorce Vueltas (3) & 767355 & 6109760 & 4,0 & 3,6 \\
\hline & Lo Ramírez (12) & 764039 & 6103898 & 47,0 & 46,3 \\
\hline & Macal (13) & 762410 & 6105157 & 14,8 & 15,1 \\
\hline \multirow[t]{3}{*}{ Constitución } & Coipué (4) & 761097 & 6093445 & 2,5 & 2,6 \\
\hline & Agua Buena (1) & 759775 & 6092747 & 1,4 & 1,5 \\
\hline & Quivolgo (14) & 752318 & 6079125 & 5,9 & 5,2 \\
\hline \multirow[t]{6}{*}{ Empedrado } & E1 Fin (7) & 740477 & 6053868 & 9,4 & 10,2 \\
\hline & El Porvenir (8) & 740115 & 6047970 & 52,0 & 43,2 \\
\hline & La Montaña (11) & 744059 & 6049591 & 62,7 & 62,8 \\
\hline & El Desprecio (6) & 742210 & 6049148 & 64,8 & 39,2 \\
\hline & Suc. Espinoza (15) & 741323 & 6050500 & 0,4 & 0,7 \\
\hline & Bellavista (2) & 739438 & 6049174 & 9,9 & 7,0 \\
\hline \multirow[t]{2}{*}{ Chanco } & La Bodega (10) & 727172 & 6036655 & 2,7 & 2,7 \\
\hline & El Corte (5) & 724415 & 6032089 & 68,2 & 66,6 \\
\hline Total & & & & 356,3 & 314,4 \\
\hline
\end{tabular}
/ Location and temporal distribution of the forest of Nothofagus alessandrii in the years 1991 and 2008 (Santelices et al., 2012).

*Datum WGS1984 18 sur

TABLA 2. Rangos de dNBR tipo de severidad esperada (Key \& Benson 2006). / Ranges of dNBR expected severity (Key \& Benson 2006).

\begin{tabular}{c|c}
\hline VALORES DEL DNBR & SEVERIDAD DEL DAÑo \\
\hline$<-0,25$ a 0,1 & Sin afectación o baja probabilidad de afectación \\
0,1 a 0,27 & Severidad baja \\
0,27 a 0,44 & Severidad moderada \\
0,4 a 0,66 & Severidad moderada a alta \\
$>0,66$ & Severidad alta \\
\hline
\end{tabular}


Mediante la comparación entre los índices obtenidos antes y después del incendio, es posible generar una composición (ráster) que luego es clasificada según la escala propuesta por Key \& Benson (2006) (Tabla 2). Para los efectos de este trabajo, las imágenes satelitales utilizadas en el análisis de severidad corresponden al sensor Sentinel II, de tamaño de pixel 10 y $20 \mathrm{~m}$, obtenidas antes y después del incendio (19.01.2017 a las $14: 45$ h y 18.02 .2017 a las 14:45 $\mathrm{h}$, respectivamente).
A

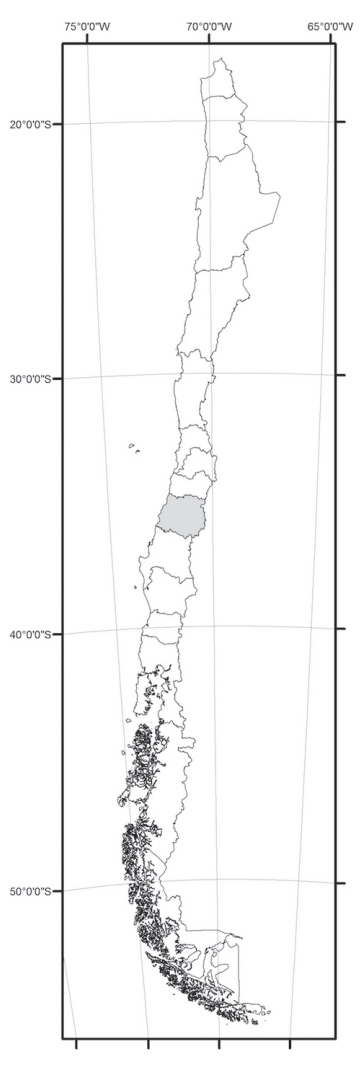

B

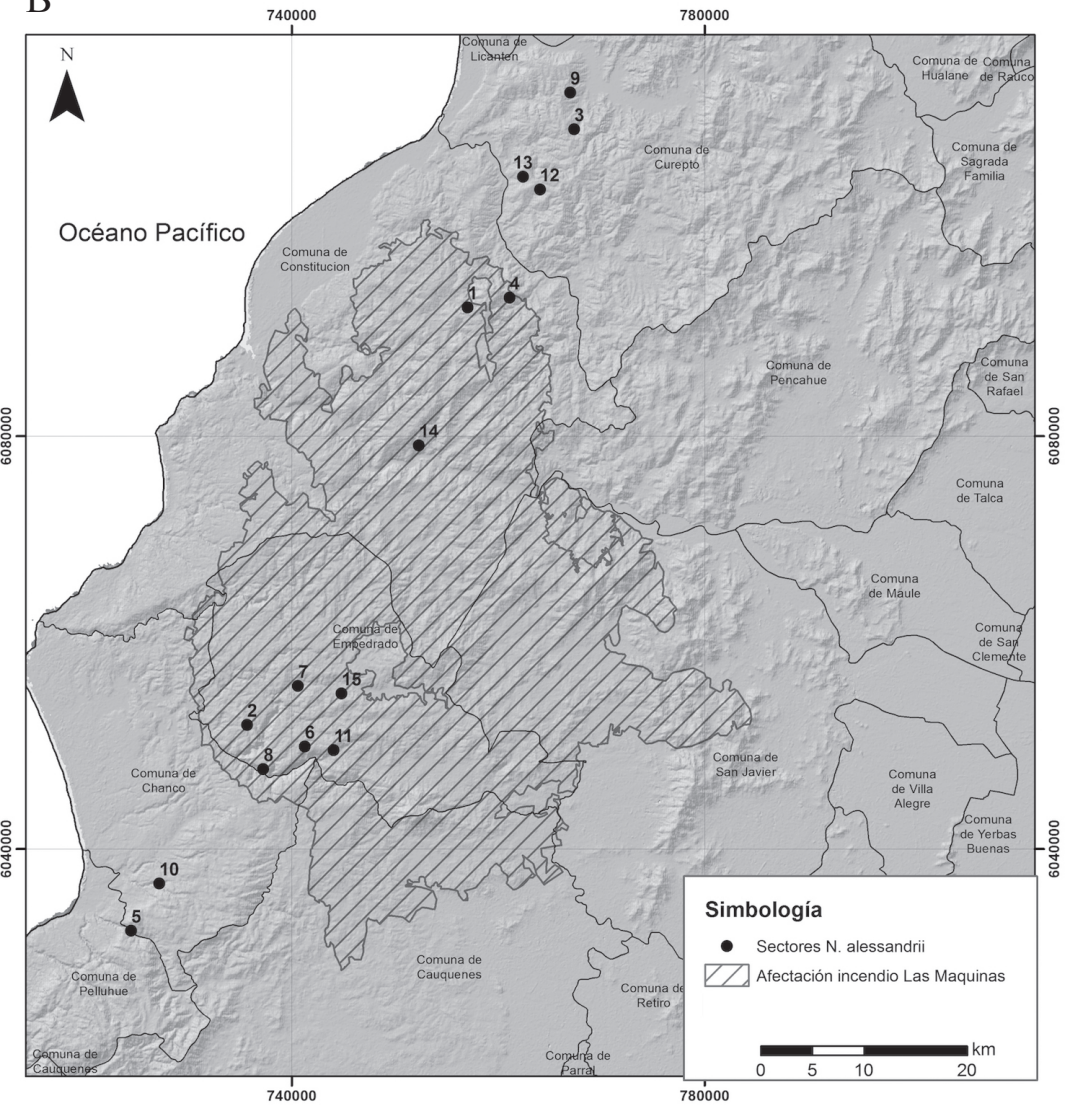

Figura 1. Distribución espacial de los bosques de Nothofagus alessandrii y superficie afectada por el incendio forestal denominado Las Máquinas en la Región del Maule en el periodo estival 2017. / Spatial distribution of Nothofagus alessandrii forests and area affected by the "Las Maquinas" forest fire in the Maule Region in the summer period 2017.

TABla 3. Superficie (ha) afectada de acuerdo a la magnitud causada por los incendios del año 2017 en los bosquetes de Nothofagus alessandrii. / Area (ha) affected according to the magnitude caused by the fires of 2017 in the forests of Nothofagus alessandrii.

\begin{tabular}{|c|c|c|c|c|c|c|}
\hline SECTOR & Sin AFECTACIÓN* & SEVERIDAD BAJA & $\begin{array}{l}\text { SEVERIDAD } \\
\text { MODERADA }\end{array}$ & $\begin{array}{c}\text { SEVERIDAD } \\
\text { MODERADA A ALTA }\end{array}$ & SEVERIDAD ALTA & TOTAL \\
\hline Coipué (4) & - & 0,17 & 0,65 & 0,93 & 0,8 & 2,55 \\
\hline Agua Buena (1) & - & 0,67 & 0,46 & 0,08 & 0,25 & 1,46 \\
\hline Quivolgo (14) & - & - & 0,05 & 0,46 & 4,66 & 5,18 \\
\hline El Fin (7) & 0,32 & 9,29 & 0,51 & 0,03 & - & 10,16 \\
\hline El Porvenir (8) & 0,34 & 15,7 & 19,54 & 5,42 & 2,15 & 43,15 \\
\hline La Montaña (11) & 0,03 & 0,18 & 5,69 & 20 & 36,88 & 62,77 \\
\hline El Desprecio (6) & 0,03 & 5,57 & 17,69 & 9,97 & 5,94 & 39,21 \\
\hline Suc. Espinoza (15) & 0,03 & 0,08 & 0,21 & 0,33 & 0,08 & 0,72 \\
\hline Bellavista (2) & 0,19 & 4,01 & 1,96 & 0,64 & 0,17 & 6,96 \\
\hline Total (ha)( \%) & $0,94(0,54 \%)$ & $35,67(20,7 \%)$ & $46,77(27,2 \%)$ & $37,86(22,0 \%)$ & $50,92(29,6 \%)$ & 172,16 \\
\hline
\end{tabular}

* Baja probabilidad de afectación $(<-0,1 \mathrm{dNBR})$ 
Comparando la información de severidad satelital con la localización de los rodales de $N$. alessandrii (Tabla 3), se observa que un $\sim 55 \%$ de la superficie de los bosques de la especie fue afectada por el fuego, concentrándose en 9 de los 15 sectores señalados en la Fig. 1. De las 172 ha afectadas por el fuego, un $\sim 52 \%$ fue clasificada como de severidad alta y severidad media alta (un $28 \%$ del total de los bosques de ruil). Sólo los bosquetes localizados en las distribuciones más septentrionales y meridionales (comunas de Curepto y Chanco) no fueron afectados por los incendios.

Si se considera que $N$. alessandrii tendría una dinámica regenerativa por claros, es decir, el autorreemplazo se produce después de que se origina un disturbio que genera un espacio dentro del bosque con mayor luminosidad, pero con sombra que ejerce una cierta protección lateral (Donoso 1993), se podría esperar que en aquellos bosques en que la severidad del daño fue alta y moderadamente alta (daño a nivel de copas), la recuperación del sistema sería más compleja. Por una parte, no habría semillas para la regeneración por monte alto $\mathrm{y}$, por otra, los rebrotes de la regeneración por monte bajo estarían expuestos a una luminosidad completa, sin una protección lateral. Adicionalmente, en forma posterior al incendio se generan condiciones propicias para el establecimiento de $P$. radiata, una de las principales amenazas que tiene la especie, y de otras especies con potencial como Genista monspessulana (L.) L. Johnson. No obstante, ello debe ser verificado en terreno.

\section{AGRADECIMIENTOS}

Se agradece el apoyo del comité de restauración de la Gerencia de Áreas Silvestres, Comité ampliado de restauración del Reserva Nacional "Los Ruiles". A los profesionales de la CONAF de la Región del Maule Sres. Alexis Villa y Felipe Barrios. Al Dr. Fabian Fassnacht del Karlsruher Institut für Technologie (KIT) por sus consejos en el procesamiento de las imágenes satelitales, a la Sra. Úrsula Partarrieu y a los revisores anónimos por sus valiosas correcciones.

\section{REFERENCIAS}

Barstow, M., Echeverría, C., Baldwin, H., Rivers, M.C. 2017. Nothofagus alessandrii. The IUCN Red List of Threatened Species 2017: e.T32033A2808995. URL: http://www. iucnredlist.org/details/32033/0. Accedido: Enero 8, 2018.
Bustamante, R., Grez, A. 1995. Consecuencias ecológicas de la fragmentación de los bosques nativos. Ambiente y Desarrollo 11(2): 58-63.

Bustamante, R.O., Castor, C. 1998. The decline of an endangered temperate ecosystem: the ruil (Nothofagus alessandrii) forest in central Chile. Biodiversity and Conservation 7(12): 1607-1626.

CONAF. 2017. Análisis del impacto de los incendios forestales ocurridos en enero y febrero de 2017 sobre los ecosistemas naturales presentes entre las regiones de Coquimbo y Los Ríos de Chile. Informe Técnico. Santiago, Chile. 50 pp.

De la Barrera, F., Ruiz, V. 2017. Evaluación del impacto de los incendios de Chile Centro-Sur en el verano del año 2017. Primera entrega. International Association of Landscape Ecology (IALE) - Chile. Chile. 6 pp.

Donoso, C. 1993. Bosques templados de Chile y Argentina. Variación, estructura y dinámica. Editorial Universitaria. Santiago, Chile. 484 pp.

Escuin, S., Navarro, R., Fernandez, P. 2008. Fire severity assessment by using NBR (Normalized Burn Ratio) and NDVI (Normalized Difference Vegetation Index) derived from LANDSAT TM/ETM images. International Journal of Remote Sensing 29(4): 1053-1073.

Keeley, J.E. 2009. Fire intensity, fire severity and burn severity: a brief review and suggested usage. International Journal of Wildland Fire 18(1): 116-126.

Key, C., Benson, N. 2006. Landscape Assessment (LA). General Technical Report RMRS-GTR-164-CD. En: Lutes, D., Keane, R., Carati, J., Key, C., Benson, N., Gangi, L. (eds.), FIREMON: Fire Effects Monitoring and Inventory System, pp. 1-55. USDA Forest Service, Rocky Mountains Research Station. Fort Collins, CO.

Miller, J.D., Thode, A.E. 2007. Quantifying burn severity in a heterogeneous landscape with a relative version of the delta Normalized Burn Ratio (dNBR). Remote Sensing of Environment 109(1): 66-80.

Myers, N., Mittermeier, R.A., Mittermeier, C.G., Da Fonseca, G.A.B., Kent, J. 2000. Biodiversity hotspots for conservation priorities. Nature 403(6772): 853-858.

Navarro, G., Caballero, I., Silva, G., Parra, P.C., Vazquez, A., CAldeIra, R. 2017. Evaluation of forest fire on Madeira Island using Sentinel-2A MSI imagery. International Journal of Applied Earth Observation and Geoinformation 58(1): 97-106.

Olivares, P., San Martín, J., Santelices, R. 2005. Ruil (Nothofagus alessandrii): Estado del conocimiento y desafíos para su conservación. Comisión Nacional del Medioambiente (CONAMA). Talca, Chile. 55 pp.

Santelices, R., Drake, F., Mena, C., Ordenes, R., NavarroCerrillo, R.M. 2012. Current and potential distribution areas for Nothofagus alessandrii, an endangered tree species from central Chile. Ciencia e Investigación Agraria 39(3): 521-531.

Recibido: 11.05 .2017

Aceptado: 24.03.2018 